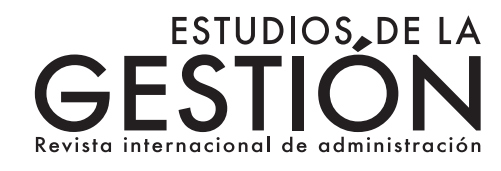

\title{
Modernización de la gestión pública y rendición de cuentas: análisis del caso ecuatoriano
}

Modernization of Public Management and Accountability:
Analysis of the Ecuadorian Case

Modernização da gestão pública e accountability: análise do caso equatoriano

\author{
Milton A. Freire Briones \\ Ministerio de Salud Pública (Ecuador) \\ mafreireb@gmail.com
}

DOI: https://doi.org/10.32719/25506641.2019.5.6

Fecha de presentación: 18 de diciembre de 2018•• Fecha de aceptación: 18 de febrero de 2019 Artículo de investigación

Licencia Creative Commons

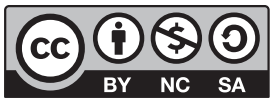




\section{Resumen}

Se busca entender por qué la modernización de la administración pública contribuye a mejorar las condiciones para la rendición de cuentas en Ecuador. Se usó el marco analítico de diseño de políticas, el rastreo de procesos con lógica bayesiana, y el planteamiento de un mecanismo causal desde la configuración de la agenda de política, la formulación de la política pública, el cambio institucional, la coordinación y adaptación de las instituciones existentes, hasta la mejora de las condiciones para la rendición de cuentas. La verificación del mecanismo causal arroja una confianza del $98,8 \%$, con lo cual se concluye que en el caso ecuatoriano existe evidencia para afirmar que la modernización de la administración pública contribuyó a mejorar las condiciones para la rendición de cuentas.

Palabras clave: Modernización, administración pública, gestión pública, rendición de cuentas. JEL: H11 Estructura, ámbito y funcionamiento del sector público.

\section{Abstract}

The goal of this article is to understand why the modernization of public administration contributes to improving the conditions for the rendering of accounts in Ecuador. The research uses the analytical framework for policy design, the process traicing with Bayesian logic, and the approach of a causal mechanism that goes from policy agenda configuration, through the formulation of public policy, the institutional change, the coordination and adaptation of existing institutions to the improvement of accountability conditions. The verification of the causal mechanism shows a confidence of $98.8 \%$, which concludes that in Ecuador there is evidence to affirm that the modernization of public administration had contributed to improving accountability conditions.

Keywords: modernization, public administration, public management, rendering of accounts. JEL: H11 Structure, scope and functioning of the public sector.

\section{Resumo}

O objetivo deste artigo é entender por que a modernização da administração pública contribui para melhorar as condições de prestação de contas no Eduador. Para responder a essa preocupação, a estrutura analítica para o desenho de políticas e rastreamento de processos com a lógica Bayesiana será usada. A discussão aborda um mecanismo causal desde a configuração da agenda política, la formulação de políticas públicas, a conseqüente mudança institucional, a coordenação e adaptação de instituições existentes para a melhoria de as condições de prestação de contas. A verificação do mecanismo causal mostra uma confiança de $98,8 \%$, o que conclui que no caso equatoriano há evidências para afirmar que a modernização da administração pública contribuiu para melhorar as condições de responsabilização.

Palavras-chave: Modernização, administração pública, gestão pública, prestação de contas. JEL: H11 Estrutura, âmbito e funcionamento do setor público. 


\section{Introducción:}

\section{¿Por qué modernizar la administración pública?}

L

a modernización de la administración pública en la región tiene dos olas. La primera, de carácter neoliberal, surge en la segunda mitad del siglo $\mathrm{XX}$, y responde a la necesidad de establecer un modelo mixto de gobernanza que se encuentra entre los modelos ideales de: gobernanza sin gobierno y gobernanza liberal-democrática. Esta ola sostiene que el accionar del Estado entorpece el mercado por cuanto, mientras menos roles juegue la administración pública, esta sería mejor para la sociedad (Aguilar Villanueva 2008).

Ya en el siglo XXI surgen teorías, tecnologías, normas y prácticas que minimizan el cuestionamiento sobre la necesidad o importancia de la administración pública, y el énfasis se da a los procesos de modernización, reforma, reinvención, reingeniería, restructuración y/o innovación (Aguilar Villanueva 2008). Así surge la segunda ola, posneoliberal, la cual también responde a un modelo de gobernanza mixto que tiene elementos de los modelos de gobernanza: liberal-democrático y estatista.

La presencia del modelo de gobernanza liberal-democrático en ambas olas deja en evidencia la necesidad de entender la modernización de la administración pública desde un enfoque neoinstitucional, es decir, como un proceso social y no como un simple resultado.

El modelo de gobernanza liberal-democrático tiene como elemento de evaluación central la rendición de cuentas (Pierre y Petes 2005). En ese sentido, un proceso de modernización puede ser calificado de más o menos exitoso, en términos de las condiciones favorables generadas para la rendición de cuentas, favoreciendo así los controles democráticos. 
El proceso de modernización se inspira en la Carta Iberoamericana de la Calidad de la Gestión Pública, ${ }^{1}$ que marca la pauta sobre el entendimiento de la calidad y la excelencia en la gestión pública en la región, trazando la hoja de ruta para las políticas públicas de modernización y mejora de las administraciones públicas; la Carta plantea nueve ejes y catorce principios para materializar y guiar una gestión pública de calidad centrada en el ciudadano (CLAD 2008).

Hay dos tipos de rendición de cuentas: vertical y horizontal. La rendición de cuentas vertical se refiere a la capacidad del soberano para, mediante el voto, elegido a sus representantes y delegar el poder. Incluso tiene la capacidad de ser electo y asumir la responsabilidad y el poder para tomar acciones en función del bien común.

La rendición de cuentas horizontal puede ser entendida como la interacción para el control mutuo, siendo así una categoría muy amplia que abarca muchos tipos de interacciones interestatales. Es aquí que varias instituciones del gobierno empiezan a jugar un rol protagónico para la rendición de cuentas, ya que buscan prevenir, cancelar, reparar y/o castigar acciones (o eventualmente inacciones) de otra agencia estatal que se presumen ilegales, tanto bajo los supuestos de transgresión como de corrupción (O'Donnell 2001).

La rendición de cuentas horizontal se caracteriza por tener instituciones de balance y de accountability horizontal asignada. Las instituciones de accountability horizontal asignada dan insumos (información) para que se pueda dar una accountability societal vertical, que no es votar-como consideraba la accountability vertical-(O'Donnell 2001).

El rol de las agencias asignadas en la accountability es fundamental ya que estas diseñan mecanismos que dan acceso a información a varios niveles de las instituciones a su cargo. Esta información pública empieza a circular libremente y puede ser utilizada por aquellos que están ejerciendo accountability vertical. La disponibilidad pública de esta información es un efecto secundario, no necesariamente planeado, del funcionamiento de instituciones de balance y asignadas. Este hecho es el que contribuye a mejorar las condi-

1. Aprobada por la X Conferencia Iberoamericana de Ministros de Administración Pública y Reforma del Estado en San Salvador el 26 y 27 de junio de 2008, y adoptada por la XVIII Cumbre Iberoamericana de Jefes de Estado y de Gobierno en San Salvador el 31 de octubre de 2008 . 
ciones para la rendición de cuentas fomentando la disponibilidad de fuentes de información alternativas que Robert Dahl correctamente enumera como una de las características centrales de una democracia política, o poliarquía (O’Donnell 2001).

Es así como las instituciones de accountability horizontal asignada juegan un rol fundamental para fomentar los controles democráticos y una consecuente mejora de las condiciones para la rendición de cuentas. Es decir, una externalidad positiva de estas instituciones es su capacidad de solicitar información que posteriormente es usada para procesos de transparencia y rendición de cuentas, aunque inicialmente tenga un carácter más reservado.

Este artículo desarrolla la relación entre la política de modernización de la administración pública, que apalanca el cambio en el modelo de gobernanza posneoliberal en Ecuador -segunda ola-, y la mejora en las condiciones para la rendición de cuentas, gracias a las externalidades positivas generadas por la participación de las instituciones de accountability horizontal asignada. El análisis se realiza a partir del año 2007 cuando inicia el gobierno de Rafael Correa en Ecuador.

\section{Metodología}

La metodología utilizada para la presente investigación es la siguiente: en una primera instancia se define un mecanismo causal, en el marco del cual se rastrea el proceso mediante el cual la definición de una política pública de modernización de la administración pública (disparador) contribuye posteriormente a mejorar las condiciones para la rendición de cuentas (resultado). El mecanismo causal define entidades, o etapas, cuya relación lógica da cuenta de la vinculación entre el disparador y el resultado.

Con la finalidad de conocer si las entidades del mecanismo causal existen, es necesario encontrar evidencia, la cual en el campo de investigación de las políticas públicas puede entenderse como instrumentos de política que dan fe de la existencia de la misma, así como de su posterior implementación. Esa evidencia es analizada con mecanismos bayesianos para obtener un valor de confianza del mecanismo causal planteado; para esto se usa mecanismos bayesianos. 
La hipótesis se sustenta en un mecanismo causal, que tiene como disparador la configuración de la agenda de política en la que el gobierno apunta a mejorar la calidad/eficiencia de la gestión pública a través de la modernización de la administración pública (HT); y, en una segunda instancia, el gobierno formula una nueva política de gestión pública basada en la modernización de la administración pública (HA). Posteriormente, el gobierno asegura la nueva política a través de un cambio institucional (HB), lo cual demanda un proceso de coordinación y adaptación de las instituciones existentes. A continuación, la interacción política entre los actores involucrados da como resultado una competencia entre los sujetos de la política para ser reconocidos como casos ejemplares (HC). Esta competencia surge en términos de cuál institución es la que ejerce una mejor gestión pública frente a las demás, dando finalmente como resultado que la nueva política mejora las condiciones para la accountability (HO).

\section{Marco teórico}

\section{Diseño de política}

El modelo de gobernanza hace referencia a las formas o modos de gobernar que involucran a actores no estatales (Levi-Faur 2012). Cada modelo de gobernanza demanda un tipo específico de administración pública que se consolida a través de cambios enmarcados en una política de modernización de la administración pública. Este proceso demanda una gobernanza jerárquica que se somete a los actores a la voluntad del gobierno, garantizando la autoridad del Estado (Kooiman 2002).

El hecho de que los modelos de gobernanza no sean ciento por ciento puros en la realidad ocasiona que los procesos de modernización de la administración pública tengan sus particularidades. En el caso ecuatoriano, los instrumentos desarrollados para modernizar la administración pública buscaban apalancar elementos de dos modelos de gobernanza distintos, el estatista y el liberal democrático; esto hace que el diseño de la política se enfrente a inconsistencia frente a los objetivos propios de cada modelo de gobernanza 
involucrado y a la falta de complementariedad de los instrumentos desarrollados por los tomadores de decisiones en el marco de la política.

Por otra parte, los intereses de los actores involucrados hacen que aparezcan instituciones formales -reglas y normas- a lo largo del proceso de implementación que dificultan el proceso de implementación de la política y generan brechas de implementación con un abanico de resultados que pueden ser calificados como más o menos exitosos; para entenderlas es necesario estudiar el proceso de la implementación desde un enfoque de causalidad (Pressman y Wildavski 1998).

El mecanismo causal, de cómo la modernización de la administración pública contribuye a mejorar las condiciones para la rendición de cuentas, resulta de la combinación de instrumentos que apalancan un modelo de gobernanza determinado. Estos instrumentos se pueden agrupar en cuatro categorías, de acuerdo con los recursos de nodalidad, autoridad, tesoro y organización del Estado (Hood 2007).

Es así que el marco analítico de la presente investigación es el diseño de políticas, el cual da vital importancia a los instrumentos de política analizando la consistencia entre fines y medios, considerando que la mezcla de instrumentos, ${ }^{2}$ seleccionados con base en el interés de los actores, permite la consecución de un resultado de política pública (Fontaine 2015).

La definición o selección de los instrumentos de política puede darse de una forma más racional y funcional a los fines buscados o de una forma más ideológica según el interés de los actores. Autores como Hood $(2007,137)$ sostienen que "la elección de instrumentos de política y formas de organización irónicamente a menudo resulta ser mucho más ideológica y políticamente disputada que las declaraciones sobre el propósito básico del gobierno".

El enfoque analítico de diseño de políticas define el denominado espacio de política pública y, en función de esta caracterización, basada en el número de programas y agencias involucradas, genera una tipología de espacios de política pública, siendo un espacio simple aquel en donde hay implicados un solo programa y una sola agencia; un espacio complejo cuando hay múlti-

2. "La teoría de análisis de instrumentos ha pasado de un estudio de instrumentos individuales a un estudio de una mezcla de instrumentos. Esta evolución abre la puerta para profundizar en estudios referentes a la selección de instrumentos y cómo evaluar la mezcla de instrumentos seleccionada" (Howlett, Kim y Weaver 2011, 130). 
ples programas y múltiples agencias; e interburocrático cuando hay un solo programa y múltiples agencias (Fontaine 2015). En el caso ecuatoriano, el espacio de la política de modernización es inicialmente un espacio complejo que durante la implementación tiende a transformarse en interburocrático debido a las adaptaciones y cambios institucionales que promovieron la consolidación de un solo programa.

\section{Rastreo de procesos basada en la lógica bayesiana}

Cuando se piensa en las políticas públicas desde su concepción hasta su posterior evaluación, pasando obviamente por la implementación, se encuentran con momentos que determinan la tendencia y los resultados de dicha política pública. Para entender qué pasó en esos momentos, cómo se concatenan esos momentos hasta llegar al resultado, es necesario abrir la caja negra de la política (Gerring 2007).

Cuando se pregunta ¿por qué la política pública de modernización de la administración pública contribuye a mejorar las condiciones para la rendición de cuentas?, surgen respuestas combinadas de la estructura y el contexto social e institucional con la agencia individual y la toma de decisiones (Bennet y Checkel 2012). La búsqueda de estas explicaciones, ligadas a abrir la caja negra de las políticas, ha generado que la explicación causal, a través de la referencia a mecanismos causales hipotéticos, tome especial relevancia para entender cualquier proceso de cambio, detrás del cual hay causas y factores complejos (Bennet y Checkel 2012).

El establecimiento de un mecanismo causal contribuye a la identificación de entidades que clarifican la relación entre el detonante y el resultado que se identifican en la pregunta de investigación. De esta forma, el rastreo de procesos permite investigar la causalidad entre variaciones de $\mathrm{X}$ y variaciones de $\mathrm{Y}$ a través de dicho mecanismo causal para posteriormente probarlo a partir de evidencias con mecanismos bayesianos (Beach y Pedersen 2016; George y Bennett 2005) para tener mayor certeza de las conclusiones del investigador. La fuerza de las inferencias que podemos hacer depende de la fuerza de la prueba empírica, junto con la precisión de la evidencia reunida (Beach y Pedersen 2016) que garantizan que nuestros conceptos teóricos estén presentes en el caso seleccionado. 
En conclusión, el mecanismo causal consiste en la definición de entidades o partes entrelazadas de un proceso (A, B, C, $n$ ) que vinculan un desencadenante (T) a un resultado (O) (Beach y Pedersen 2013). Las entidades no necesariamente corresponden a una secuencia de eventos históricos, ya que cada entidad intermedia puede surgir de diferentes procesos, a base de un patrón de cambio institucional por estratificación (Mahoney y Thelen 2010).

Es necesario encontrar un caso típico, es decir, en el que exista tanto el disparador como el resultado que se espera encontrar para el mecanismo causal. El caso ecuatoriano, al ser un caso típico, permitirá evidenciar cómo funcionó el mecanismo causal desde la variante centrada en la teoría, por lo que se espera aclarar cómo X (disparador) contribuyó a ocasionar Y (resultado) (Beach y Pedersen 2016; George y Bennett 2005). Para esto se planteó un diseño de pruebas empíricas que aíslen el funcionamiento de mecanismos particulares (Beach y Pedersen 2016).

En este contexto, el teorema de Bayes proporciona una manera formal de evaluar el significado que confirma o no las observaciones empíricas y discute su calidad como evidencia (Fontaine, Narváez y Fuentes 2018).

Las pruebas para validar las evidencias del mecanismo causal se basan en los niveles de certeza y exclusividad (Bennett 2015; Van Evera 1997). La certeza se define como la probabilidad de encontrar una evidencia dada una hipótesis $\mathrm{p}(\mathrm{e} \mid \mathrm{h})$; en otras palabras, es la probabilidad de encontrar la evidencia cuando la teoría planteada es verdadera. La exclusividad es la probabilidad de encontrar la misma evidencia si la hipótesis o teoría es falsa $\mathrm{p}$ (e $\mid \neg$ h) (Van Evera 1997). La descripción detallada se aprecia en el anexo 1. De esta manera se usan las probabilidades para garantizar la validez o no del mecanismo causal planteado.

\section{a) Pruebas de certeza y exclusividad}

Para las pruebas de certeza y exclusividad se considera que la probabilidad de que una hipótesis sea verdades $\mathrm{p}(\mathrm{h})$, es $50 \%$ lo que genera una igual probabilidad de corroborar o no la hipótesis, lo cual da más consistencia al análisis posterior. Esto implica que la probabilidad de que no se cumpla la hipótesis es del mismo valor. 
Para corroborar la existencia de una hipótesis se pueden usar cuatro tipos de pruebas: paja en el viento, aro, pistola humeante y doblemente decisiva. La prueba de paja en el viento se caracteriza por tener una baja probabilidad de encontrar una evidencia tanto si existe la teoría como si no; por eso, se asumen valores aleatorios tomados en experiencias previas de 0,4 y 0,3 respectivamente (Fontaine, Narváez y Fuentes 2018). Por otra parte, las pruebas doblemente decisivas se caracterizan porque la evidencia tiene una alta probabilidad de existir si la teoría es verdadera y una baja probabilidad de existir si la teoría es falsa, por lo que estudios previos asignan valores de 0,9 y 0,1 respectivamente a estas probabilidades (Fontaine, Narváez y Fuentes 2018).

Entre las dos pruebas expuestas en el párrafo anterior están las pruebas de aro y prueba de pistola humeante. La prueba de aro tiene como característica que la probabilidad de encontrar la evidencia, cuando una teoría es verdadera, es mayor que en la prueba de pistola humeante, pero, a su vez, la probabilidad de que en una prueba de aro exista evidencia cuando la teoría no es verdadera es mayor a la que se puede tener en una prueba de pistola humeante. A continuación, se presenta una tipología de las pruebas empíricas utilizadas para el diseño de la investigación (Fontaine, Narváez y Fuentes 2018) (ver tabla 1).

Para realizar una de estas pruebas, por ejemplo, la de aro, es necesario la aplicación de esta a todos los instrumentos de una política, es decir, los de nodalidad, autoridad, tesoro y organización, para cada uno de los cuales se han definido evidencias. Se considera una alta certeza cuando se obtiene una probabilidad de que exista la evidencia y exista la entidad analizada de $90 \%$ $(\mathrm{p}(\mathrm{e} \mid \mathrm{h})=0,9)$ y una baja exclusividad cuando la probabilidad de que exista la evidencia, pero no se cumpla la entidad analizada del $10 \%(\mathrm{p}(\mathrm{e} \mid \neg \mathrm{h})=0,1)$ (Fontaine, Narváez y Fuentes 2018).

Cuando se consideran de manera separada, la prueba de aro se puede presentar el valor de certeza más bajo que equivale a $25 \%(\mathrm{C}=0,25)$; pero el valor de desconfirmación más alto puede tomar el valor de $-37,5 \%(\mathrm{C}=$ $-0,375)$; si se considera toda la tipología de instrumentos, la certeza podría incrementar al 98,8\%, con lo que una teoría se confirmaría, incluso si para uno de los cuatro tipos de instrumento la prueba es negativa el nivel de confianza llegaría al 79,4\%, como se puede ver en las tablas 2 y 3 (Fontaine, Narváez y Fuentes 2018). 
Tabla 1

Tipos de pruebas empíricas en el marco del bayesianismo lógico

\begin{tabular}{|c|c|c|c|c|c|c|c|c|c|c|}
\hline $\begin{array}{l}\text { Tipo de } \\
\text { prueba }\end{array}$ & $P(h)$ & $P(-h)$ & $\mathrm{P}(\mathrm{e} / \mathrm{h})$ & $P(-e / h)$ & $\mathrm{P}(\mathrm{e} / \mathrm{h})$ & $P(-e /-h)$ & $\mathrm{P}(\mathrm{h} / \mathrm{e})$ & $\mathrm{P}(\mathrm{h} /-e)$ & $\begin{array}{l}C 1=P(h / \\
e)-(P(h)\end{array}$ & $\begin{array}{l}\text { C2: } P(h /- \\
\text { e)-P(h) }\end{array}$ \\
\hline $\begin{array}{l}\text { Paja en el } \\
\text { viento }\end{array}$ & 0,5 & 0,5 & 0,4 & 0,6 & 0,3 & 0,7 & 0,57 & 0,46 & 0,07 & $\begin{array}{l}-0,04 \\
\end{array}$ \\
\hline Aro & 0,5 & 0,5 & 0,9 & 0,1 & 0,3 & 0,7 & 0,75 & 0,13 & 0,25 & $-0,38$ \\
\hline $\begin{array}{l}\text { Pistola } \\
\text { humeante }\end{array}$ & 0,5 & 0,5 & 0,4 & 0,6 & 0,1 & 0,9 & 0,8 & 0,4 & 0,3 & $-0,1$ \\
\hline $\begin{array}{l}\text { Doblemente } \\
\text { decisiva }\end{array}$ & 0,5 & 0,5 & 0,9 & 0,1 & 0,1 & 0,9 & 0,9 & 0,1 & 0,4 & $-0,4$ \\
\hline
\end{tabular}

$P(h): \quad$ Probabilidad de encontrar la evidencia esperada.

$\mathrm{P}(-\mathrm{h})$ : $\quad$ Probabilidad de no encontrar la evidencia esperada.

$\mathrm{P}(\mathrm{e} / \mathrm{h})$ : Probabilidad de encontrar la evidencia cuando se cumple la hipótesis.

$\mathrm{P}(-\mathrm{e} / \mathrm{h})$ : Probabilidad de no encontrar la evidencia cuando se cumple la hipótesis.

$\mathrm{P}(\mathrm{e} / \mathrm{h})$ : Probabilidad de encontrar la evidencia cuando no se cumple la hipótesis.

$\mathrm{P}(-\mathrm{e} / \mathrm{h})$ : Probabilidad de no encontrar la evidencia cuando no se cumple la hipótesis.

$\mathrm{P}(\mathrm{h} / \mathrm{e})$ : Probabilidad de encontrar la hipotesis cuando hay la evidencia. Se calcula así:

$$
\frac{\mathrm{P}(h) \mathrm{P}(\mathrm{e} / \mathrm{h})}{\mathrm{P}(h) \mathrm{P}(\mathrm{e} / \mathrm{h})+\mathrm{P}(-\mathrm{h}) \mathrm{P}(\mathrm{e} / \mathrm{-h})}
$$

$\mathrm{P}(\mathrm{h} / \mathrm{-e})$ : Probabilidad de que se cumpla la hipotesis pese a no existir evidencia. Se calcula así:

$$
\frac{P(h) P(-e / h)}{P(h) P(-e / h)+P(-h) P(-e /-h)}
$$

C1 y C2: Son los valores de confianza.

Fuente: Fontaine, Narváez y Fuentes (2018).

Elaboración propia.

Por lo tanto, esta investigación identifica los instrumentos de política inicialmente planteado y su posterior cambio por adaptación o calibración, cuya combinación resultó en el resultado de la política, es decir la mejora de las condiciones para la rendición de cuentas en Ecuador. 
Tabla 2

Certeza y exclusividad cuando se cumple la existencia de evidencia de los 4 instrumentos

\begin{tabular}{|c|c|c|c|c|c|c|c|c|c|c|c|}
\hline $\begin{array}{c}\text { Evidencial } \\
\text { instrumento }\end{array}$ & Cumple & $\mathrm{P}(\mathrm{h})$ & $P(-h)$ & $\mathrm{P}(\mathrm{e} / \mathrm{h})$ & $P(-e / h)$ & $P(e /-h)$ & $P(-e /-h)$ & $\mathrm{P}(\mathrm{h} / \mathrm{e})$ & $P(h /-e)$ & $\begin{array}{c}\mathrm{C} 1= \\
\mathrm{P}(\mathrm{h} / \mathrm{e})- \\
\mathrm{P}(\mathrm{h})\end{array}$ & $\begin{array}{c}C 2=P(h /- \\
\text { e) }-P(h)\end{array}$ \\
\hline & & 0,500 & 0,500 & 0,900 & 0,100 & 0,300 & 0,700 & 0,750 & 0,125 & 0,250 & ט, \\
\hline Autoridad & Cumple & 0,750 & 0,250 & 0,900 & 0,100 & 0,300 & 0,700 & 0,900 & 0,300 & 0,150 & 0,450 \\
\hline Tesoro & Cumple & 0,900 & 0,100 & 0,900 & 0,100 & 0,300 & 0,700 & 0,964 & 0,563 & 0,064 & $-0,338$ \\
\hline Organización & Cumple & 0,964 & 0,036 & 0,900 & 0,100 & 0,300 & 0,700 & 0,988 & 0,794 & 0,024 & $-0,170$ \\
\hline
\end{tabular}

Fuente: adaptado de Fontaine, Narváez y Fuentes (2018).

Elaboración propia.

\section{Tabla 3}

Certeza y exclusividad cuando se cumple la existencia de evidencia de 3 de los 4 instrumentos

\begin{tabular}{|c|c|c|c|c|c|c|c|c|c|c|c|}
\hline $\begin{array}{l}\text { Evidencial } \\
\text { instrumento }\end{array}$ & Cumple & $P(h)$ & $P(-h)$ & $\mathrm{P}(\mathrm{e} / \mathrm{h})$ & $\mathrm{P}(-\mathrm{e} / \mathrm{h})$ & $P(e /-h)$ & $P(-e /-h)$ & $\mathrm{P}(\mathrm{h} / \mathrm{e})$ & $\mathrm{P}(\mathrm{h} /-\mathrm{e})$ & $\begin{array}{c}\mathrm{C} 1= \\
\mathrm{p}(\mathrm{h} / \mathrm{e})- \\
\mathrm{p}(\mathrm{h})\end{array}$ & $\begin{array}{c}C 2=p(h /- \\
e)-p(h)\end{array}$ \\
\hline Nodalidad & Cumple & 0,500 & 0,500 & 0,900 & 0,100 & 0,300 & 0,700 & 0,750 & 0,125 & 0,250 & $-0,375$ \\
\hline Autoridad & No cumple & 0,750 & 0,250 & 0,900 & 0,100 & 0,300 & 0,700 & 0,900 & 0,300 & 0,150 & $-0,450$ \\
\hline Tesoro & Cumple & 0,300 & 0,700 & 0,900 & 0,100 & 0,300 & 0,700 & 0,563 & 0,058 & 0,263 & $-0,242$ \\
\hline Organización & Cumple & 0,563 & 0,438 & 0,900 & 0,100 & 0,300 & 0,700 & 0,794 & 0,155 & 0,232 & $-0,407$ \\
\hline
\end{tabular}

Fuente: adaptado de Fontaine, Narváez y Fuentes (2018).

Elaboración propia.

\section{Descripción del mecanismo causal}

A continuación, se presenta el mecanismo causal teórico que se busca corroborar con la presente investigación (ver tabla 4). 


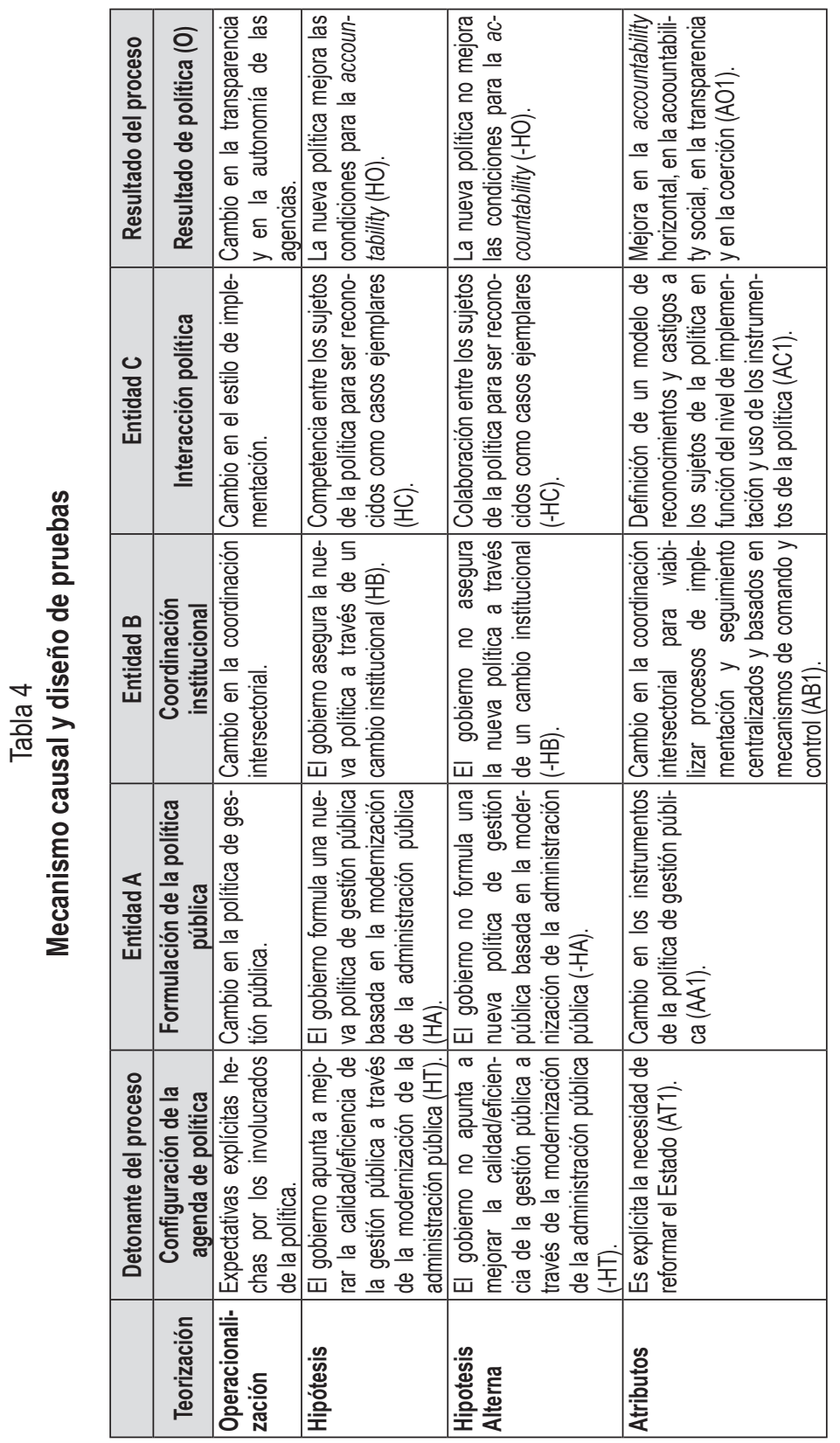




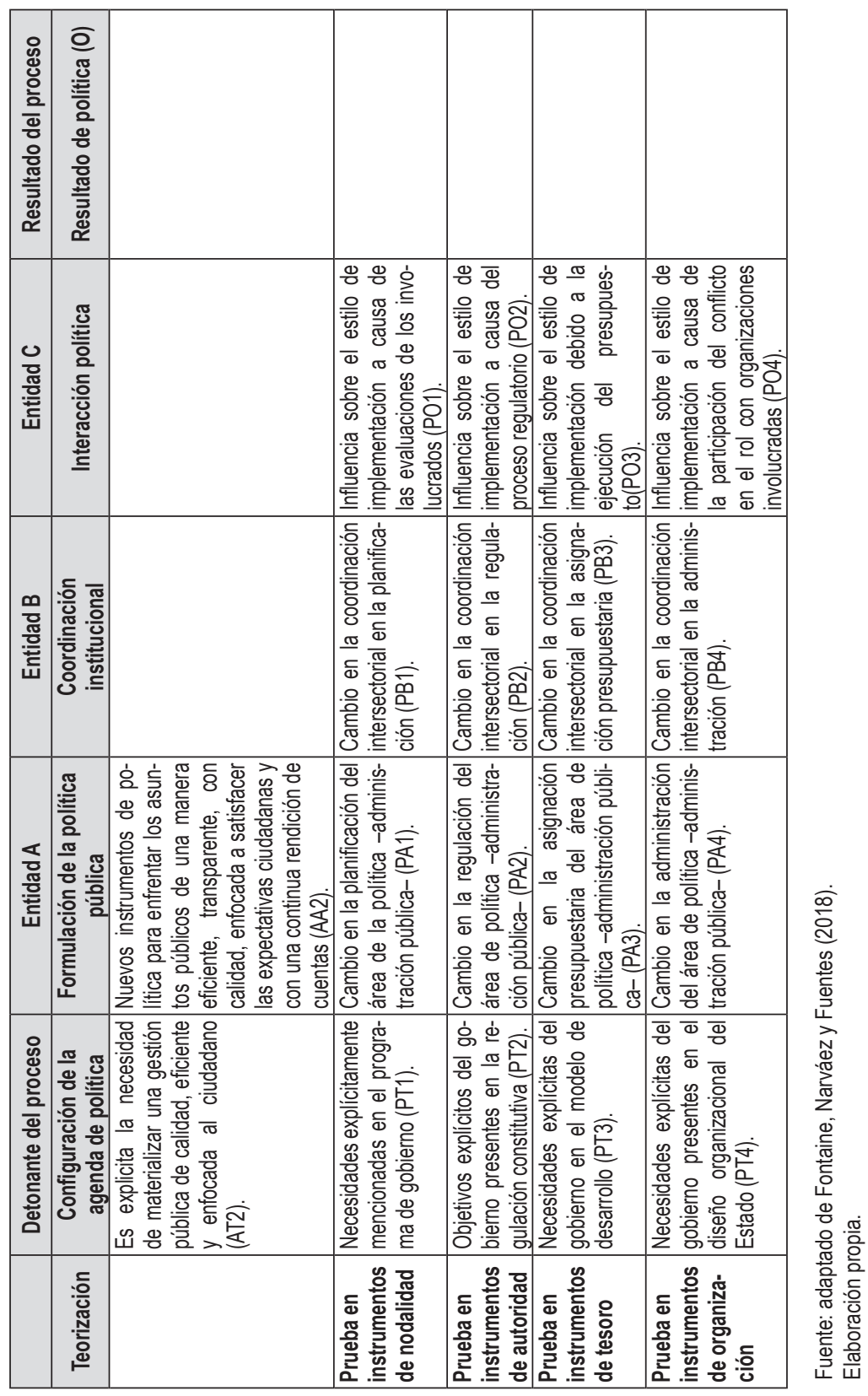


Las pruebas para el resultado de la política son las siguientes:

Tabla 5

Diseño de pruebas para la comprobación del resultado de política

\begin{tabular}{|l|l|l|}
\hline & \multicolumn{2}{|c|}{ Resultado de política } \\
\hline \multirow{4}{*}{ Policy mix } & Accountability horizontal & $\begin{array}{l}\text { Cambio en el equilibrio y control de la autonomía } \\
\text { de las agencias. }\end{array}$ \\
\cline { 2 - 3 } & Accountability social & Cambio en el control social sobre la política. \\
\cline { 2 - 3 } & Transparencia & Cambio en el acceso a información. \\
\cline { 2 - 3 } & Coersión & Cambio en las sanciones para prácticas ilegales. \\
\hline
\end{tabular}

Elaboración propia.

b) Mecanismo causal de como la modernización de la administración pública contribuye a la rendición de cuentas

El mecanismo causal que explica la relación existente entre la modernización de la administración pública y la mejora de las condiciones para la se operativiza de la siguiente manera:

Inicialmente el gobierno apunta a mejorar la calidad y eficiencia de la gestión pública a través de la modernización de la administración pública (CLAD 2008), por lo que hizo explícitas las expectativas de los involucrados de la política de reformar el Estado (AT1) y la necesidad de materializar una gestión pública de calidad, eficiente y enfocada al ciudadano (AT2).

Posteriormente, el gobierno formula una nueva política de gestión pública basada en la modernización de la administración pública, lo cual se da a través de un cambio en la política de gestión pública. Esto permite pasar de la primera a la segunda ola de modernización, dejando de lado la visión de un Estado moderno en el marco de la nueva gestión pública a un Estado moderno en el marco de la gobernanza (Aguilar Villanueva 2008). En ese sentido, se tienen los siguientes atributos: cambio en los instrumentos de la política de gestión pública (AA1) y nuevos instrumentos de política para enfrentar los asuntos públicos de una manera eficiente, transparente, con calidad, en- 
focada a satisfacer las expectativas ciudadanas y con una continua rendición de cuentas (AA2).

Luego, el gobierno asegura la nueva política a través de un cambio institucional, lo que implica un cambio en la coordinación intersectorial, enfocado básicamente para permitir la implementación de la política. En este sentido, debe evidenciarse un proceso de implementación y coordinación que busca implantar con éxito la nueva política, con roles claramente definidos. El atributo de esta entidad es un cambio en la coordinación intersectorial para viabilizar procesos de implementación y seguimiento centralizados y basados en mecanismos de comando y control (AB1).

Después aparece una competencia entre los sujetos de la política para ser reconocidos como casos ejemplares, por ende, en la operacionalización se evidencia un cambio en el estilo de implementación.

Al homologar y estandarizarse la concepción de un Estado modelo se define un modelo de una buena administración; concomitantemente al mismo, surge una escala de madurez que permite que se pueda comparar el nivel de madurez entre instituciones. Esto genera una competencia por evidenciar mayores avances, en términos de administración más moderna, eficiente, y abre las puertas a una competencia entre los sujetos de la política que facilita el proceso de implementación.

Se diseñan y generan rankings de cumplimiento de los componentes de la política de modernización de la administración pública y se diseña un esquema o modelo de reconocimiento, adaptado a las necesidades propias de la implementación. Adicionalmente cobran relevancia reportes de desempeño y gobierno abierto (OCDE 2015; Ramírez-Alujas y Naser 2014; Dunleavy y Hood 1994; Belbis 2013). Esto genera evidencias que se van haciendo más o menos públicas y que contienen información de la gestión de las distintas instituciones, lo que ocasiona una mejora en las condiciones para la rendición de cuentas a nivel horizontal, social y la coerción; las evidencias que sustentan la calificación para el reconocimiento es una información valiosa que es un insumo fundamental para ejercicios de rendición de cuentas, tanto vertical como horizontal.

El atributo de esta entidad es la definición de un modelo de reconocimientos y castigos a los sujetos de la política en función del nivel de implementación y uso de los instrumentos de la política (AC1). 


\section{c) Recolección de evidencia}

La evidencia se recolectó de información documental desde el año 2006 hasta 2017. La información recolectada sirvió como prueba para cada instrumento y para cada entidad y los resultados se resumen a continuación (ver tabla 6).

\section{Resultados}

\section{El gobierno apunta a mejorar la calidad/eficiencia de la gestión pública a través de la modernización de la administración pública (HT)}

El Plan de Gobierno de Alianza País, divulgado en 2006, antes de que Correa ganara las elecciones de 2007, estipula la necesidad de reestructurar y fortalecer las entidades del Estado con el objeto de satisfacer las exigencias de una sociedad moderna que requiere eficiencia y efectividad, transparencia y mayor participación ciudadana para defender el bien público e impulsar el desarrollo. También se plantea la necesidad de desconcentrar la gestión y desprivatizar el Estado para mejorar la calidad de la administración pública y ganar en términos de transparencia (Alianza PAIS 2006).

Como evidencia de instrumentos de autoridad se espera observar que existen objetivos explícitos en la regulación constitutiva $\left(\mathrm{P}_{\mathrm{T} 2}\right)$, es decir en la Constitución (EC 2008), como instrumento que pone especial énfasis en la calidad de la prestación de servicios públicos; definiéndose los principios del servicio público, de los cuales destaco: eficacia, eficiencia, calidad, desconcentración, descentralización, participación, transparencia y evaluación (EC 2008). Se hace referencia a la necesidad de reformar el Estado para la consecución de estos fines.

En el Plan Nacional de Desarrollo (PND 2007-2011) se encuentran instrumentos de tesoro; el objetivo 12 del PND 2007-2011 indica "Reformar el Estado para el bienestar colectivo" (Senplades 2007, 278) y se hace referencia explícita a que es necesario recuperar el Estado, desprivatizarlo y reformarlo, siendo evidente la existencia de los dos atributos esperados. 


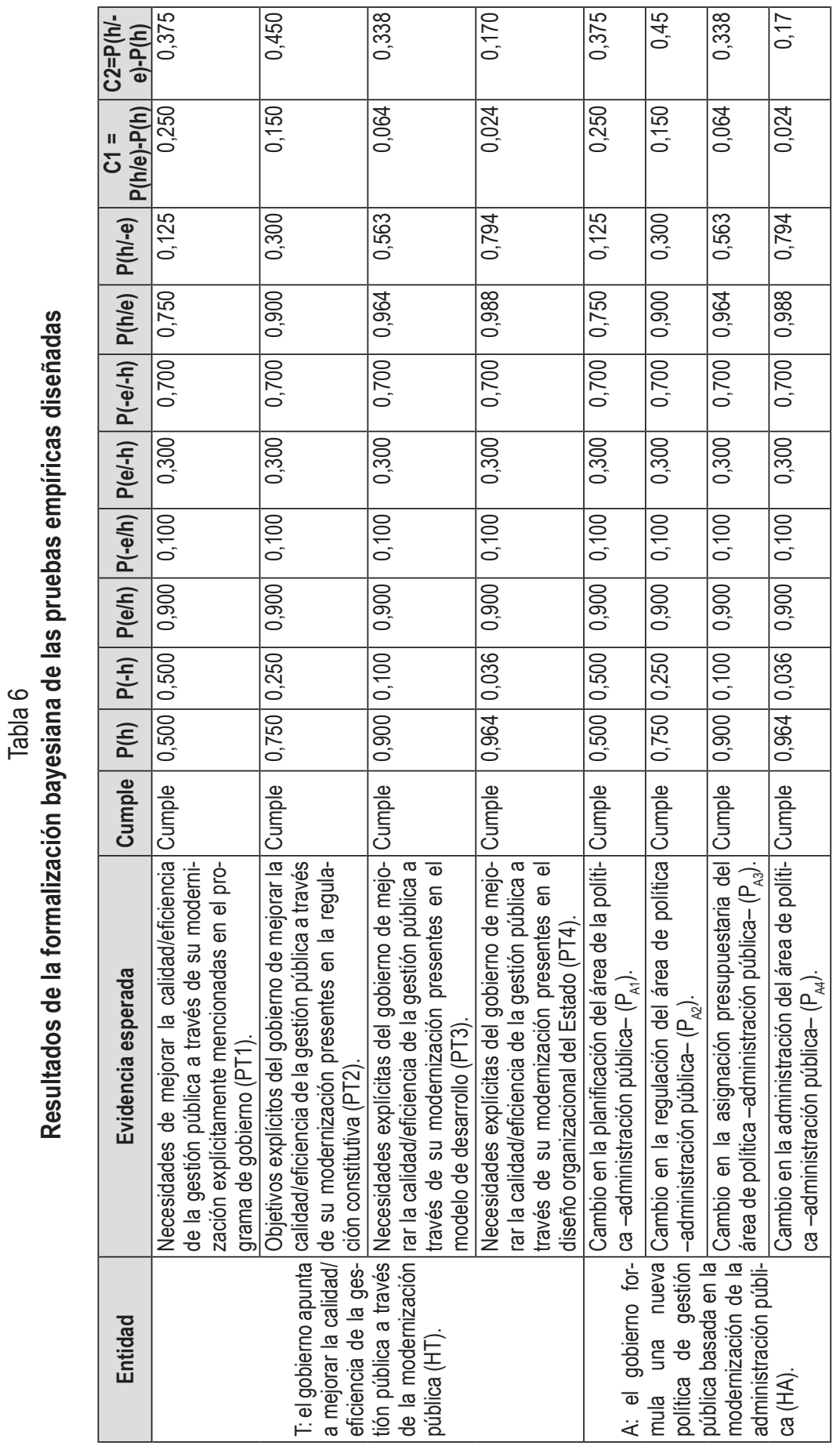




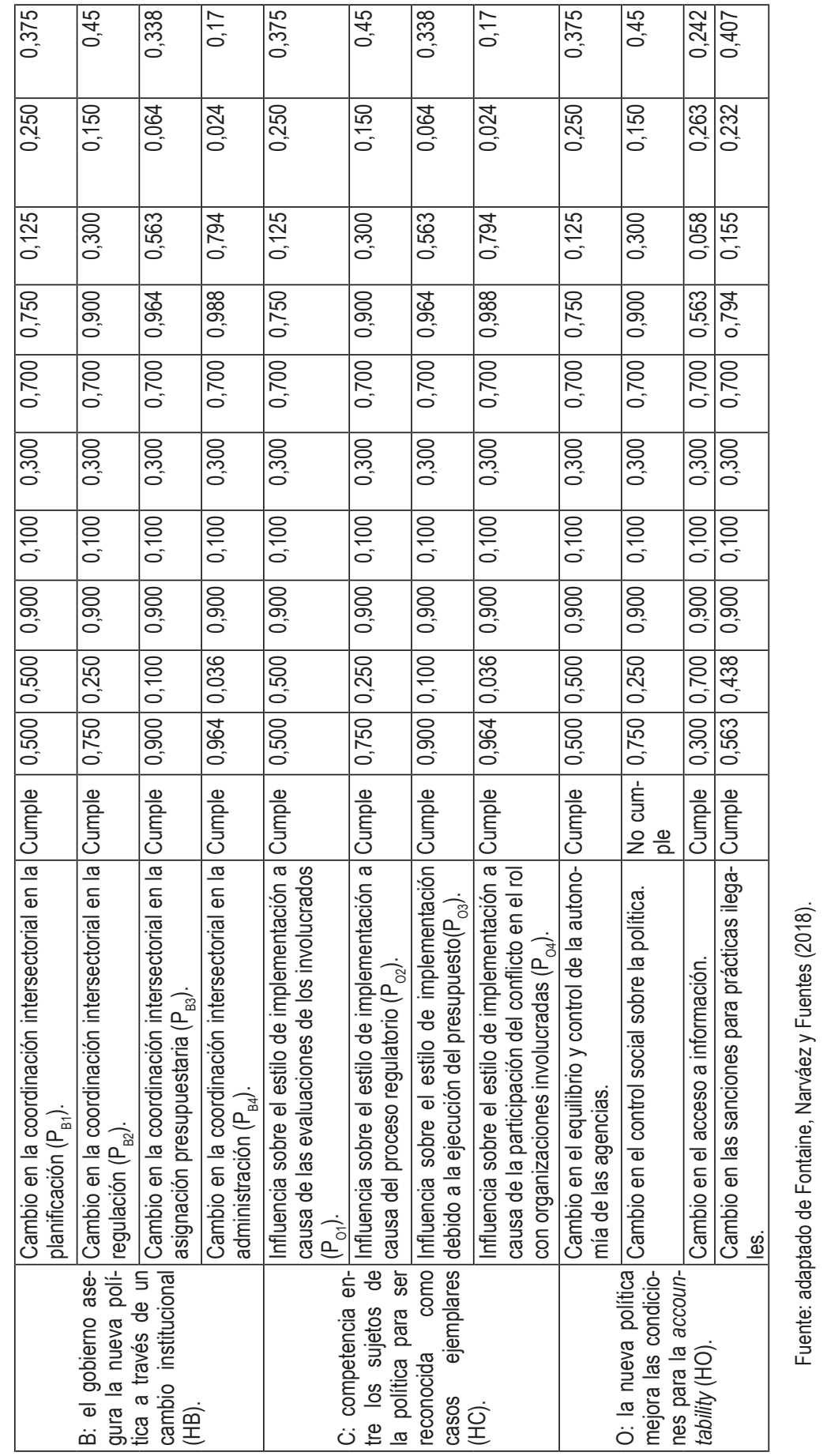


Finalmente, en los instrumentos de organización se espera observar explícitamente las expectativas de los involucrados de la política de reformar el Estado (AT1); y la necesidad de materializar una gestión pública de calidad, eficiente y enfocada al ciudadano (AT2); estando esto presente en el diseño organizacional del Estado (PT4), es decir, por una parte que tengan responsables claros y, por otra parte, que se considere la necesidad de un cambio institucional, dado que este es en sí mismo AT1. Como evidencia se destaca el Decreto Ejecutivo 103 (EC 2007) que fusiona el Consejo Nacional de Modernización (CONAM) y la Secretaría Nacional de los Objetivos de Desarrollo del Milenio (SODEM) a la Secretaría Nacional de Planificación y Desarrollo (Senplades) (EC 2007). De esta manera la Senplades es la responsable de la modernización de la administración pública definiendo explícitamente la responsabilidad de la modernización de la administración pública.

Al haberse encontrado las cuatro evidencias esperadas en el detonante, con sus respectivos atributos, se puede decir que hay una confianza del 98 , $8 \%$ de que esta entidad del mecanismo causal existe.

\section{El gobierno formula una nueva política de gestión pública basada en la modernización de la administración pública}

El Plan Nacional de Desarrollo 2009-2013 surge luego de las reformas constituyentes del año 2008 y plantea desafíos como el de "reconstruir lo público" (Senplades 2009, 11). El objetivo 12 de este Plan es "Construir un Estado democrático para el Buen Vivir", siendo explícita la necesidad de re-construir, re-definir o reformar el Estado. Este proceso demanda "un servicio civil eficiente, meritocrático y orientado por resultados" (Senplades $2009,353)$. Este plan hace referencia a la necesidad de promover/cambiar instrumentos como la desconcentración del Ejecutivo y la descentralización hacia los gobiernos autónomos descentralizados, con la finalidad de materializar una "gestión pública eficiente vinculada a la gente" (Senplades 2009, 354). Todo este proceso de reforma busca la recuperación de la capacidad estatal bajo esquemas de gestión eficiente, transparente, descentralizado, desconcentrado y participativo, a fin de conseguir una mayor coherencia funcional y legitimidad democrática en sus intervenciones (Senplades 2009). A lo largo del plan se evidencian políticas que hacen referencia a una nueva 
organización y rediseño institucional del Estado, el fomento de un servicio público eficiente y competente, gestión de servicios públicos con calidad, desarrollo de alianzas público-privadas, diseño de una arquitectura nacional de información que posibilite a los ciudadanos obtener provecho de los servicios estatales provistos a través de las tecnologías de la información y comunicación, gobierno electrónico, mejorar en la relación e integración de las entidades del sector público mediante el apoyo de herramientas tecnológicas de la información y comunicación para lograr la simplificación de trámites y reducir requisitos, nuevo esquema estatal de regulación y control sobre los servicios y bienes públicos, entre otros (Senplades 2009).

Es evidente que este nuevo Plan Nacional de Desarrollo abre las puertas a cambio en los instrumentos de la política de gestión pública (AA1) y nuevos instrumentos de política para enfrentar los asuntos públicos de una manera eficiente, transparente, con calidad, enfocada a satisfacer las expectativas ciudadanas y con una continua rendición de cuentas (AA2).

Respecto al cambio en la regulación del área de política de administración pública es necesario hacer referencia a la Ley Orgánica del Servicio Pública (LOSEP), que tiene como objetivo el desarrollo profesional, técnico y personal de los servidores públicos, para lograr el permanente mejoramiento, eficiencia, eficacia, calidad, productividad del Estado y de sus instituciones, mediante la conformación, el funcionamiento y el desarrollo de un sistema de gestión del talento humano (EC 2010). Adicionalmente define roles de rectoría, regulación, implementación, evaluación y control en el marco de la política de modernización para las entidades públicas como el Ministerio del Trabajo (MDT), la Secretaría Nacional de Administración Pública (SNAP) y la Senplades. Adicionalmente define nuevos instrumentos como: el sistema de talento humano con sus subsistemas; la certificación de calidad con las necesidades de evaluar el cumplimiento de metas institucionales, la remuneración variable, entre otros (Asamblea Nacional 2010). De esta manera la LOSEP promueve un cambio en los instrumentos de la política de gestión pública (AA1) y plasma nuevos instrumentos de política para enfrentar los asuntos públicos de una manera eficiente, transparente, con calidad, enfocada a satisfacer las expectativas ciudadanas y con una continua rendición de cuentas (AA2). 
Respecto al cambio en la asignación presupuestaria del área de política de administración pública $\left(\mathrm{P}_{\mathrm{A} 3}\right)$, se identifican programas y proyectos nuevos, que surgen a raíz de la nueva política. Como evidencia de esto se han encontrado las siguientes evidencias: Decreto Ejecutivo 555 (2010) y el Perfil del Programa de Reforma Institucional de la Gestión Pública (2011). En el Decreto Ejecutivo 555 se establece la implementación de gobierno por resultados (GPR) en la Función Ejecutiva -entendido como un proyecto de inversión que canaliza recursos- . Este proyecto era crucial para el gobierno para modernizar la administración pública y, posteriormente, convertirla en una fuente de información para la gestión presupuestaria, la rendición de cuentas y la remuneración variable. Por otra parte, la SNAP encabezó las conversaciones con el BID para conseguir recursos que permitan la mejora de la administración pública; es así que surge el perfil del proyecto "Programa de Reforma Institucional de la Gestión Pública". En el documento del perfil se hace referencia a instrumentos como la matriz de competencias, el Código Orgánico de Planificación y Finanzas Públicas y la Ley Orgánica del Servicio Público, que permiten una consolidación normativa y la unificación de los roles rectores de planificación participativa, finanzas públicas, control de la gestión y servicio público en la Senplades, el Ministerio de Finanzas, SNAP y MDT. Para la ejecución de esta estrategia el gobierno determinó la creación de un comité de los órganos rectores denominado Comité de Gestión Pública Institucional, que tiene a su cargo coordinar, articular, dirigir, y emitir políticas y lineamientos estratégicos respecto de la implementación de la restructuración de la gestión pública institucional y en ese marco prioriza la asignación de recursos económicos hacia diversas iniciativas gubernamentales (BID 2011; Reyes 2011).

Finalmente, respecto al cambio en la administración del área de política analizada (administración pública) $\left(\mathrm{P}_{\mathrm{A} A}\right)$, es evidente la definición y establecimiento de nuevos roles, tanto para la Senplades, SNAP y MDT, que forman parte del denominado "Comité de Gestión Pública Institucional" (Presidencia de la República del Ecuador 2011). La SNAP juega un rol protagónico de coordinación por lo que fue necesario reformar la secretaría mediante el Acuerdo Ministerial 571 de 2011 que se crea la Subsecretaría de Innovación y Gestión Estratégica, que lidera el direccionamiento de las instituciones del Gobierno en temas de eficiencia, eficacia e innovación para la administración pública (Secretaría Nacional de la Administración Pública 2011). En el 
mismo Acuerdo Ministerial se crea la Subsecretaría de Seguimiento, Control y Calidad, encargada de realizar el control y seguimiento de la gestión cotidiana de los procesos y proyectos de la administración pública, así como del control de la calidad de la gestión de los mismos y a su vez genera acciones correctivas y propuestas para la eficiente y oportuna toma de decisiones, además de propender a lograr un desempeño de excelencia de la gestión de la administración pública a través de la calidad. También se crea la Subsecretaría de Tecnologías de la Información, que contribuirá con la transformación y modernización de la gestión pública, a través de la ejecución, soporte, operación y normalización de los proyectos y procesos de innovación tecnológica que sean definidos y priorizados previamente por el Secretario Nacional de la Administración Pública (Secretaría Nacional de la Administración Pública 2011). De esta manera es evidente la existencia de una nueva institucionalidad encaminada a implementar la nueva política.

Al haberse encontrado las cuatro evidencias esperadas en el disparador, con sus respectivos atributos, se puede concluir que hay una confianza del $98,8 \%$ de que este elemento del mecanismo causal existe.

\section{El gobierno asegura la nueva política a través de un cambio institucional (HB)}

Como instrumento de nodalidad se creó el Comité de Gestión Pública Interinstitucional con la finalidad de "coordinar la implementación del modelo de reestructuración de la gestión pública institucional en la función ejecutiva y mantener un solo direccionamiento estratégico interinstitucional, garantizando una gestión pública sustentada en los principios de la administración pública establecidos en la Constitución y enfocada en el cambio de la cultura institucional y la satisfacción del ciudadano" (Presidencia de la República del Ecuador 2011). En el Acuerdo Ministerial 571 se establece que la SNAP aprobará proyectos tecnológicos en varios ámbitos de la mejora de gestión. Si a esto sumamos que esta entidad preside el Comité de Gestión Pública Institucional, que operativizará el "Programa de Reforma Institucional de la Gestión Pública", que es una operación de más de US \$ 200 millones de dólares con el BID, podemos apreciar cómo se genera un instrumento claro de modalidad y tesoro que cambia la coordinación intersectorial para viabilizar 
la implementación y seguimiento centralizados basados en mecanismos de comando y control.

Se evidencia un rol protagónico y centralizador de la SNAP, en distintos proyectos de mejora de gestión. Por ejemplo, la implementación de Gobierno por Resultados (Presidencia de la República del Ecuador 2010); la promoción de la gestión por procesos y servicios, con un enfoque de calidad (Secretaría Nacional de la Administración Pública 2013); y en la gestión documental y archivo (Secretaría Nacional de la Administración Pública 2015). Adicionalmente el Decreto Ejecutivo 439 (EC 2011, 3), en las disposiciones generales, establece que "Ninguna entidad de la Función Ejecutiva podrá iniciar un proceso de reestructura paralelo al modelo propuesto por el Comité, y cualquier contratación que se realice o pretenda realizar al respecto deberá ser comunicada y aprobada previamente por el Comité". Esto corrobora que existió un cambio en la coordinación intersectorial para viabilizar procesos de implementación y seguimiento centralizados y basados en mecanismos de comando y control (AB1).

Respecto al cambio en la coordinación intersectorial en la asignación presupuestaria $\left(\mathrm{P}_{\mathrm{B} 3}\right)$ se toma como evidencia el contrato de la operación de crédito 2653/OC-EC con el BID (2012). Esta operación tiene dos componentes que financian los nuevos instrumentos mencionados anteriormente en el marco de la política de modernización de la administración pública (BID 2012; República del Ecuador 2011). El Comité de Gestión Pública que administra esta operación evidencia un cambio en la coordinación intersectorial para viabilizar procesos de implementación y seguimiento centralizados y basados en mecanismos de comando y control (AB1).

Finalmente, respecto al cambio en la coordinación intersectorial en la administración $\left(\mathrm{P}_{\mathrm{B} 4}\right)$, se evidencia en el art. 3 del Decreto Ejecutivo 439 (EC 2011, 5) que "El Comité de Gestión Pública Interinstitucional estará conformado por: a) El Secretario Nacional de la Administración Pública, quien lo presidirá; El Secretario o Secretaria Nacional de Planificación y Desarrollo; y, El Ministro o Ministra de Relaciones Laborales" (Presidencia de la República del Ecuador 2011), con lo que se cambió la coordinación intersectorial para viabilizar procesos de implementación y seguimiento centralizados y basados en mecanismos de comando y control (AB1). 
Al haberse encontrado las cuatro evidencias esperadas en el disparador, con sus respectivos atributos, podemos decir que hay una confianza del $98,8 \%$ de que este elemento del mecanismo causal existe.

\section{Competencia entre los sujetos de la política para ser reconocidos como casos ejemplares $(\mathrm{HC})$}

Respecto a cómo la evaluación y participación de los involucrados en la política influyen en el estilo de implementación (PC1) se espera evidenciar mecanismos de evaluación del avance e impacto de la implementación que no surgen como tal desde el inicio de la implementación, sino que son resultado de una evolución fruto de la adaptación y calibración en el proceso de implementación. De esta manera esta evidencia observable contribuye a dar fe de la existencia del atributo de esta entidad que es definición de un modelo de reconocimientos y castigos a los sujetos de la política en función del nivel de implementación y uso de los instrumentos de la política (AC1).

Para evidenciar esto hemos recurrido a la Guía Metodológica de Aplicación del Modelo Ecuatoriano de Excelencia, expedida mediante Resolución SCG-DNGC-001 (EC 2016). Esta guía es un elemento que surge por la necesidad de articular todos los procesos existentes en un marco de implementación que facilite el establecimiento de estándares y reconocimientos nacionalmente aplicados. Adicionalmente este instrumento evidencia el rol coordinador y articulador que venía desempeñando la SNAP.

Respecto a la existencia de influencia sobre el estilo de implementación a causa del proceso regulatorio (PO2) se puede apreciar, en el año 2012, que el MDT tiene un rol más protagónico en la política y es así que expide el Acuerdo Ministerial 55 que norma la ejecución del proceso de evaluación de la conformidad. En el caso de la información producto de la evaluación realizada por la Senplades y SNAP, sus resultados serán remitidos al MDT, para ser condensados y procesados de manera integral en el informe final de evaluación de la conformidad (Ministerio de Trabajo 2012). De esta manera el Ministerio de Trabajo busca jugar un rol más protagónico en el proceso de reconocimiento. Esto es precedente a la Resolución SCG-DNGC-001 de la SNAP, pero si se compara estos dos instrumentos es clara la existencia de un conflicto por posicionar el instrumento de evaluación y reconocimiento. 
Así podemos observar que está presente el atributo de esta entidad que es definición de un modelo de reconocimientos y castigos a los sujetos de la política en función del nivel de implementación y uso de los instrumentos de la política (AC1).

Sobre la existencia de influencia en el estilo de implementación debido a la ejecución del presupuesto (PO3), se pueden verificar cambios en el financiamiento de los mecanismos de evaluación del avance e impacto de la implementación que no surgen como tal desde el inicio de la implementación, sino que son resultado de evolución de los mecanismos iniciales o nuevos mecanismos que surgen fruto de la adaptación y calibración en el proceso de implementación. Esto se evidencia a través del Acuerdo 366 del Ministerio de Trabajo, a través del cual se expide el Reglamento para el pago de Remuneración variable por eficiencia (EC 2012), creado en la LOSEP (Asamblea Nacional 2010) y en el art. 272 del reglamento de esta ley.

Por ende, es evidente la existencia del atributo de esta entidad que es definición de un modelo de reconocimientos y castigos a los sujetos de la política en función del nivel de implementación y uso de los instrumentos de la política (AC1).

Finalmente, respecto a la existencia de influencia sobre el estilo de implementación a causa de la participación del conflicto en el rol con organizaciones involucradas (PC4), se pueden verificar cambios en el estilo de implementación a causa de los mecanismos de evaluación del avance e impacto de la implementación que no surgen como tal desde el inicio de la implementación y que involucran la participación de varias organizaciones en los siguientes instrumentos: Acuerdo Ministerial 55 del MDT (2012); Decreto Ejecutivo 1522 (2013); Decreto Ejecutivo 3 (2013); Resolución 118 (2013); y Decreto Ejecutivo 5 (2017). En estos instrumentos se evidencia un conflicto por retomar protagonismo entre la SNAP y el MDT. Por otra parte, el Decreto Ejecutivo 1522 establece en su art. 7 la transformación de la Secretaría Nacional de Transparencia de Gestión en Subsecretaría y dispone su fusión por absorción a la SNAP (Presidencia de la República del Ecuador 2013). Esto deja en evidencia que la SNAP pasa a tener control sobre transparencia en la gestión. Como consecuencia de lo expuesto se cambian los estatutos de la Secretaría Nacional de la Administración Pública, con la finalidad de evidenciar su liderazgo en la modernización y mejora de la 
administración pública a través de la Resolución 118 de 2015 (Secretaría Nacional de la Administración Pública 2013). A partir de esta reforma la misión de la Secretaría Nacional de la Administración Pública es la de "Mejorar la eficiencia de las organizaciones del Estado Central e Institucional a través de políticas y procesos que optimizan la calidad, la transparencia y la calidez del Servicio Público".

Hasta aquí es evidente el protagonismo por el liderazgo de los procesos de mejora de la administración pública entre el MDT y la SNAP. Fruto de este conflicto podría ser el Decreto Ejecutivo 5, que es uno de los primeros del Gobierno de Lenín Moreno, que suprime la SNAP, pasando muchas de sus competencias al MDT y SENPLADES (Presidencia de la República del Ecuador 2017).

Todas estas intenciones y acciones desarrolladas a lo largo del gobierno de Rafael Correa, desde el año 2007 hasta 2017, han generado cambios en la política de modernización de la administración pública, así como un proceso de reforma del Estado, que han contribuido en la mejora de las condiciones de la rendición de cuentas. Por ejemplo, la implementación de GPR obligó a muchas instituciones a llenar oportunamente información en el sistema con firmas de responsabilidad, esa información generada para cumplir con la normativa vigente emitida por las entidades de accountability asignada como la SNAP fue utilizada en varios ejercicios de rendición de cuentas. Si a esto le sumamos el hecho de la existencia de ranking de cumplimiento en el reporte de información, podemos ver claramente las condiciones para que las instituciones generen información que, a manera de externalidad positiva, mejora las condiciones para la rendición de cuentas.

Al haberse encontrado las cuatro evidencias esperadas en el disparador, con sus respectivos atributos, podemos decir que hay una confianza del 98, $8 \%$ de que este elemento del mecanismo causal existe.

\section{La nueva política mejora las condiciones para la accountability (HO)}

Para evidenciar el resultado de la política de modernización de la administración pública, que es que la nueva política mejora las condiciones para la accountability (HO), se espera encontrar lo siguiente: cambio en el 
equilibrio y control de la autonomía de las agencias; cambio en el control social sobre la política; cambio en el acceso a información; y cambio en las sanciones para prácticas ilegales.

En esto debemos indicar que la función ejecutiva, en los que se implementó una agresiva reforma con la finalidad de mejorar la eficiencia, eficacia, calidad y transparencia de la gestión, desarrolló modelos de implementación basados en el reconocimiento de buenas prácticas, que se consolidan en la Guía del Modelo Ecuatoriano de Excelencia. Fruto de ello las instituciones han generado prácticas que contribuyen a la accountability, como, por ejemplo:

En la sección de transparencia de las páginas web se reportan metas y objetivos de las unidades administrativas, en esta sección se transparenta la planificación y los resultados de la gestión de todas las oficinas del Ejecutivo. En esta sección se carga información tomada del gobierno por resultados y se adjunta incluso las firmas de responsabilidad; esta manera de acceder a la información es más funcional a la que existía en el año 2007 cuando no existían ni si quiera firmas de responsabilidad ni fuentes sistematizadas de información. Esto tuvo mejores resultados en unos sectores que en otros, sin embargo, a nivel general se generaron mejores condiciones para la rendición de cuentas.

La facilidad de acceder a información permite a la ciudadanía y a las instancias de control ejercer control democrático; muestra de ello es que es un insumo valioso en varios exámenes especiales, contribuyendo así al equilibrio de poderes que una sociedad democrática necesita. La evidencia de esto son los exámenes de auditoría de varias instituciones que hacen referencia a información de gobierno por resultados.

Estos exámenes son los que determinan las respectivas sanciones por las distintas fallas administrativas.

\section{Conclusiones}

Implicaciones de la modernización de la administración pública para la rendición de cuentas: la confianza posterior del mecanismo causal se incre- 
menta hasta un $98,8 \%$, por lo que podemos decir que las entidades expuestas en la operacionalización de la teoría existieron en el caso ecuatoriano, por ende, la modernización de la administración pública en el caso ecuatoriano contribuyó a mejorar las condiciones para la rendición de cuentas.

Se evidencia que se posicionó desde un inicio en la agenda política la necesidad de modernizar la administración pública, lo que llevó a la formulación de una política, evidenciándose varios instrumentos que permiten su implementación.

Las evidencias nos permiten probar que el gobierno ecuatoriano formuló una nueva política pública de modernización de la gestión pública basada en la modernización de la administración pública, la cual inicialmente estaba a cargo de la Senplades y a la que se fueron añadiendo nuevos actores como la SNAP y el MDT. Esta política contaba con los instrumentos de modalidad, autoridad, tesoro y organización que garantizan en sí mismo la existencia de una política pública al respecto (Howlett, Kim y Weaver 2011).

El Gobierno ecuatoriano buscó asegurar la política pública de modernización de la gestión pública a través de cambios institucionales, en los que son de vital importancia la coordinación existente entre los actores involucrados en el denominado Comité Institucional de Gestión Pública.

Es evidente que la implementación aislada de distintas iniciativas llevó a desarrollar la denominada Guía del Modelo Ecuatoriano de Excelencia, la cual contiene un modelo que abarca buenas prácticas que se pueden conseguir, siempre y cuando se implementen los instrumentos previamente desarrollados. Este modelo no estaba inicialmente contemplado. Incluso haciendo un análisis crítico, se podría decir que esto debía formar parte del diseño inicial de la política, pero en realidad no fue así: aparece como un instrumento posterior fruto de la necesidad de articular los esfuerzos y generar un modelo de reconocimiento que genera una "sana competencia" entre las instituciones públicas.

De esta forma las instituciones de balance, Senplades, SNAP y MDT, promovieron instrumentos y un modelo que obligaba a que las instituciones generen evidencias de cumplimiento que a la postre permitieron mejorar las condiciones para la accountability.

Es importante destacar el papel de la Senplades, SNAP y MDT como organismos de accountability horizontal asignada, en cuyo rol promueven 
a que las otras instituciones del gobierno generen información útil para la rendición de cuentas. Este mismo hecho en particular evidencia la mejora de las condiciones para la rendición de cuentas.

En conclusión, un cambio en la política de modernización de la gestión pública en el Ecuador llevó a mejorar las condiciones para la accountability.

\section{Referencias}

Aguilar Villanueva, Luis. 2008. Gobernanza y gestión pública. Ciudad de México: Fondo de Cultura Económica.

Alianza País. 2006. Plan de gobierno 2007-2011. Quito: Alianza PAIS.

Banco Interamericano de Desarrollo (BID). 2011. Contrato de préstamo No. 2653/OC-EC. Quito, 15 de diciembre de 2011. Consulta: abril de 2018. 〈http://contenidos.culturaypatrimonio.gob.ec/wp-content/uploads/CONTRATO_2678_BID.pdf).

---. 2011. Perfil del Proyecto Programa de Reforma Institucional de la Gestión Pública. Perfil de Proyecto para Operación Financiera. Quito: BID.

Beach, Derek, y Rasmus Pedersen. 2013. Process Tracing Methods: Foundations and Guidelines. Michigan: University of Michigan Press.

---. 2016. "Case Selection Techniques in Process-tracing and the Implications of Taking the Study of Causal Mechanisms Seriously". Sociological Methods \& Research, No. 14: 1-35.

Belbis, Juan Ignacio. 2013: "Gobernanza abierta en espacios legislativos". En César Calderón y Sebastián Lorenzo, edits., Open Government, 239-249. Alcalá la Real: Algón Editores.

Bennett, Andrew. 2015. "Appendix: Disciplining our Conjectures: Systematizing Process Tracing With Baysian Analysis". En Andrew Bennett y Jeffrey Checkel, edits., Process Tracing: From Metaphor to Analytic Tool, 211-213. Cambridge: Cambridge University Press.

---,y Jeffrey Checkel. 2012. Process Tracing: From Philosophical Roots to Best Practices. Londres: Cambridge University Press.

Brady, Henry, y David Collier. 2010. Rethinking Inquiry: Drivers Tools, Shared Standards. Lanham: Rowman \& Littlefiel Publishers.

Centro Latinoamericano de Administración para el Desarrollo (CLAD). 2008. Carta Iberoamericana de la Calidad en la Gestión Pública. San Salvador: CLAD.

Dahl, Robert, Ian Shapiro y José Antonio Cheibub. 2003. The Democracy Source Book. Cambridge: MIT Press.

Dunleavy, Patrick, y Christopher Hood. 1994. "From Old Public Administration to New Public Management". Public Money \& Management 14, No. 3: 9-16.

EC. 2008. Constitución del República del Ecuador. Registro Oficial 449, 20 de octubre. 
Modernización de la gestión pública y rendición de cuentas: análisis del caso ecuatoriano

---. 2010. Ley Orgánica del Servicio Público. Registro Oficial 298, 6 de octubre.

EC Ministerio de Relaciones Laborales. 2011. Programa de reforma institucional de la gestión pública. Quito: Ministerio de Relaciones Laborales.

EC Ministerio de Trabajo. 2012. "Acuerdo Ministerial 366”, 3 de enero.

---. 2012. "Acuerdo Ministerial 55”, 18 de mayo.

EC Secretaría Nacional de la Administración Pública (SNAP). 2011. "Acuerdo Ministerial 571 ". 2011.

---. 2013. "Acuerdo Ministerial 1580". 13 de febrero de 2013.

---. 2013. "Resolución 118”. Quito, 2 de agosto de 2013.

---. 2015. "Acuerdo Ministerial 1043". 2 de febrero de 2015.

---. 2016. "Resolución SCG-DNGC-001". 27 de septiembre de 2016.

EC Secretaría Nacional de Planificación y Desarrollo (Senplades). 2007. Plan Nacional de Desarrollo (2007-2010). Quito: Senplades.

---. 2009. Plan Nacional de Desarrollo (2009-2013). Quito: Senplades.

Fontaine, Guillaume. 2015. El análisis de politicas públicas. Madrid: Anthropos / FLACSO.

---, Iván Narváez y José Luis Fuentes. 2018. Is Energy Transition Hindered by Resource Nationalism? A Causal Mechanism of Implementation Gap in Ecuador. Quito: FLACSO.

George, Alexander, y Andres Bennett. 2005. Case Studies and Theory Development in the Social Sciences. Cambridge: MIT Press.

Gerring, John. 2007. Case Study Research. Cambridge: Cambridge University Press.

Hood, Christopher. 2007. "Intellectual Obsolescence and Intellectual Makeovers: Reflectons on the Tools of Government after Two Decades". Gobernance 20, No. 1: 127-144.

Howlett, Michael, Jonathan Kim y Paul Weaver. 2011. “Assessing Instrument Mixes Through Program- and Agency Level Data: Methodological Issues in Contemporary Implementation Research". Designing Public Policies: Principles and Instruments 17, No. 1: 327-350.

Jackson, Patrick. 2011. The Conduct of Inquiry in International Relations. Londres: Routledge.

Kooiman, Jan. 2002. Modern Governance. New Govemment-Society Interactions. Londres: Sage.

Lascoumes, Pierre, y Patrick Le Gales. 2007. "Introduction: Understanding Public Policy through its Instruments: From the Nature of Instruments to the Sociology of Public Policy instrumentation". Gobernance 20, No. 1: 1-21.

Levi-Faur, Davis, edit. 2012. From "Big Government" to "Big Governance"? Londres: Oxford University Press.

Mahoney, James, y Kathleen Thelen. 2010. "A Theory of Gradual Institutional Change”. En de James Mahoney y Kathleen Thelen, edits., Explaining Institutional Change: Ambiguity, Agency and Power, 1-30. Nueva York: Cambridge University Press.

O’Donnell, Guillermo. 2001. “Accountability horizontal: la institucionalización legal de la desconfianza política". POSTData, No. 7: 11-34. 
Peters, Guy, Jon Pierre y Tiina Randma-Liiv. 2010. "Global Financial Crisis, Public Administration and Governance: Do New Problems Require New Solutions?". Public Organiz Rev. 11, No. 1: 13-27.

Pierre, Jon, y Guy Petes. 2005. Governing Complex Societies. Gran Bretaña: Palgrave.

Presidencia de la República del Ecuador. 2007. "Decreto Ejecutivo 103”. Registro Oficial, 22 de febrero.

---. 2010. "Decreto Ejecutivo 555". 19 de noviembre.

---. 2011. "Decreto Ejecutivo 439". 11 de abril.

---. 2013. "Decreto Ejecutivo 1522". 12 de junio.

---. 2013. "Decreto Ejecutivo 3". 13 de junio.

---. 2017. "Decreto Ejecutivo 5". 24 de mayo de 2017.

Pressman, Jeffrey, y Aaron Wildavski. 1998. Implementación: cómo grandes expectativas concebidas en Washington se frustran en Oakland. Ciudad de México: Fondo de Cultura Económica.

Ramírez-Alujas, Álvaro, y Alejandra Naser. 2014. Plan de gobierno abierto: una hoja de ruta para los gobiernos de la región. Santiago: ONU / CEPAL.

Van Evera, Stephen. 1997. Guide to Methods for Students of Political Science. Nueva York: Cornell Univesity Press. 


\section{Anexo 1: \\ Lógica bayesiana}

El grado inicial de confianza $\mathrm{p}(\mathrm{h})$ es la confianza inicial en una teoría antes de que la misma sea sometida a una prueba empírica. Una vez realizada la prueba se tiene el grado posterior de confianza, que es la probabilidad de que una teoría sea verdadera, dada la evidencia disponible $\mathrm{p}(\mathrm{h} \mid \mathrm{e})$.

Se mide por el teorema de Bayes de la siguiente manera:

$$
\mathrm{p}(\mathrm{h} \mid \mathrm{e})=\frac{\mathrm{p}(\mathrm{h}) \mathrm{p}(\mathrm{e} \mid \mathrm{h})}{\mathrm{p}(\mathrm{h}) \mathrm{p}(\mathrm{e} \mid \mathrm{h})+\mathrm{p}(\neg \mathrm{h}) \mathrm{p}(\mathrm{e} \mid \neg \mathrm{h})}
$$

Por el contrario, el grado posterior de confianza en la ausencia de evidencia de la siguiente manera:

$$
\mathrm{p}(\mathrm{h} \mid \neg \mathrm{e})=\frac{\mathrm{p}(\mathrm{h}) \mathrm{p}(\neg \mathrm{e} \mid \mathrm{h})}{\mathrm{p}(\mathrm{h}) \mathrm{p}(\neg \mathrm{e} \mid \mathrm{h})+\mathrm{p}(\neg \mathrm{h}) \mathrm{p}(\neg \mathrm{e} \mid \neg \mathrm{h})}
$$

La diferencia entre el grado posterior de confianza $\mathrm{p}(\mathrm{h} \mid \mathrm{e})$ o $\mathrm{p}(\mathrm{h} \mid \neg \mathrm{e})$ y el anterior $\mathrm{p}(\mathrm{h})$ da una actualización de la confianza en una teoría. Eso es $\mathrm{C}=\mathrm{p}(\mathrm{h} \mid \mathrm{e})-\mathrm{p}$ (h) si la prueba es positiva y

$\mathrm{C}=\mathrm{p}(\mathrm{h} \mid \neg \mathrm{e})-\mathrm{p}(\mathrm{h})$ si la prueba es negativa. Eventualmente, una teoría puede ser confirmada

si $\mathrm{C}>0$, no confirmado si $\mathrm{C}<0$, o indeterminado si $\mathrm{C}=0$. La confianza posterior del mecanismo causal está dada por el que sigue a la entidad más débil. 